\title{
Supramolecular Chirality of the Two-component Supramolecular Copolymer Gels: Who Determines the Handedness?
}

Yaqing Liu, Chunfeng Chen, Tianyu Wang, ${ }^{*}$ and Minghua Liu*

Beijing National Laboratory for Molecular Science CAS, Key Laboratory of Colloid Interface and Chemical Thermodynamics, Institute of Chemistry, Chinese Academy of Science, Beijing 100190, P. R. China.

\section{1: Experimental Section}

Materials: Tartaric acids (1a, 1b), mesomeric tartaric acid (1c), succinic acid (2), tartaric acids diester (3), enantiomeric malic acids (4a, 4b), diacetyl tartaric acid (5a, 5b) were purchased from Sigma-Aldrich and used as received. Bolaamphiphiles containing L-histidine methyl ester head-groups (BolaHis) was synthesized according to the method described in the literature. ${ }^{11}$ Milli-Q water $(18.2 \mathrm{M} \Omega \mathrm{cm})$ was used in all cases.

Instruments: Transmission electron microscopy (TEM) images were obtained on a JEM $2100 \mathrm{~F}$ operating at accelerating voltages of $200 \mathrm{kV}$. JASCO UV-550 and JASCO J-810 CD spectrometers were used for the UV/Vis and CD spectral measurements, respectively. Fourier transform infrared (FT-IR) spectra were recorded on a JASCO FT/IR-660 plus spectrophotometer with a wavenumber resolution of 4 
$\mathrm{cm}^{-1}$ at room temperature. X-ray diffraction (XRD) measurements was performed on a Rigaku D/Max-2500 X-ray diffract meter (Japan) with $\mathrm{Cu} / \mathrm{K} \alpha$ radiation $(\lambda=1.5406$ $\AA$ ), which was operated at $45 \mathrm{kV}, 100 \mathrm{~mA}$. The DSC thermograms of supramolecular copolymers were obtained using a VP-DSC microcalorimetric system (MicroCal).

Method: The supramolecular copolymers were cast onto carbon-coated $\mathrm{Cu}$ grids (unstained), and the water was evaporated at ambient conditions first, then vacuum-dried for $12 \mathrm{~h}$ for TEM measurements. The $\mathrm{KBr}$ pellets made from the vacuum-dried supramolecular copolymers were used for FT-IR spectra measurements.

The $0.1 \mathrm{~mm}$ cuvette was used for UV-Vis spectra and CD spectra measurements. The quartz-plate-sustained supramolecular copolymers films were used for X-ray diffraction (XRD) measurements.

\section{2: Supplementary Figures}
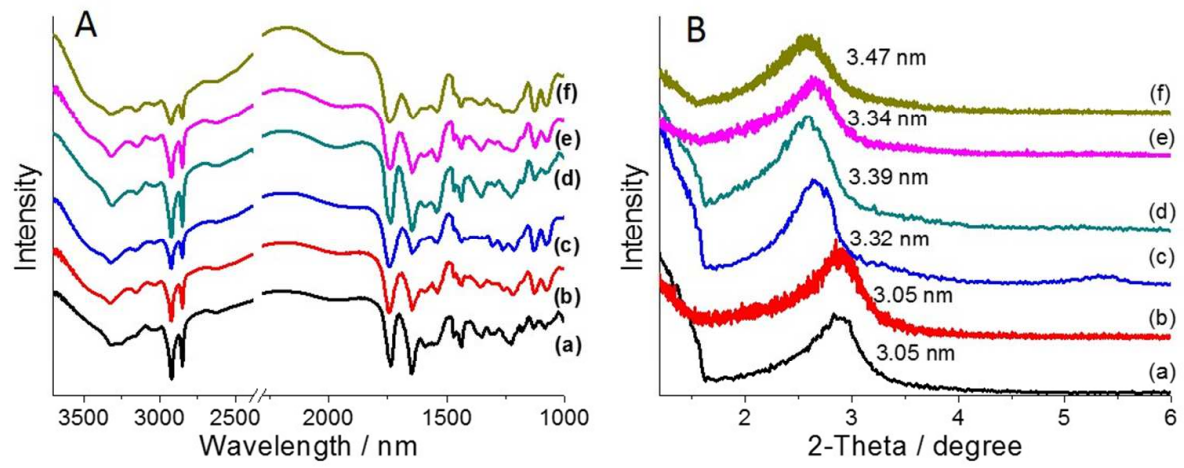

Figure S1: FT-IR spectra (A) and XRD pattern (B) of 1b/BolaHis assemblies with molar ratios equal to $1 / 1$ (a), 2/1 (b), 3/1 (c), and 1a/BolaHis assemblies with molar ratios equal to $1 / 1$ (d), $2 / 1$ (e), $3 / 1$ (f). 


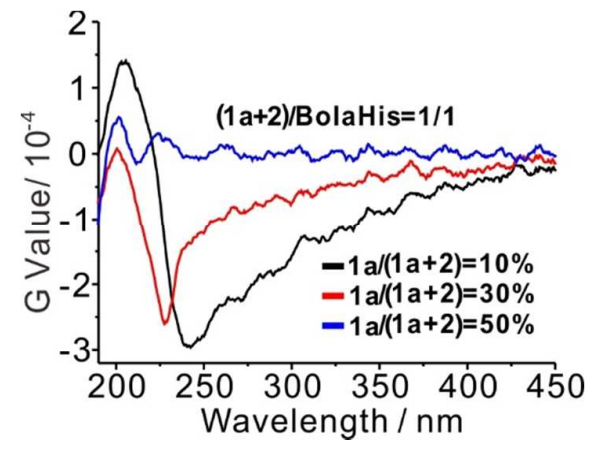

Figure S2: CD spectra of $\mathbf{1 a / 2} /$ BolaHis supramolecular gels with different molar ratios.

(A)<smiles>CCOC(=O)C(O)C(O)C(=O)OCC</smiles>

(B)

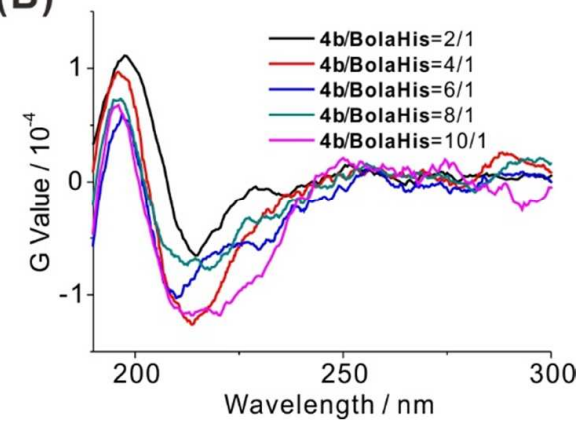

Figure S3: (A) Molecular structures of tartaric acids diester (3); (B) CD spectra of 4b/BolaHis supramolecular copolymers with different molar ratios. 